\title{
Preconcentration of morphine in urine sample using a green and solvent-free microextraction method
}

https://doi.org/10.1515/gps-2019-0023

Received October 10, 2018; accepted March 26, 2019.

Abstract: The ability of extraction and preconcentration of small amounts of substances from biological samples is very important in medical toxicology. On the other hand, minimal use of organic solvents is an important issue to prevent environmental damage. In the present study, we developed a new solid phase microextraction fiber using plant extracts as sorbent for extraction and preconcentration of morphine in urine sample. For this purpose, raw carbon nanotubes (CNTs) were functionalized with tobacco extracts. Functionalization was confirmed by Fourier transform infrared (FTIR) and Raman spectroscopy in addition to scanning electron microscope (SEM) images. The functionalized CNTs were coated on polypropylene hollow fiber. The results of HPLC analysis showed that the produced fiber could preconcentrate a very low concentration of morphine $(0.25 \mathrm{ng} / \mathrm{ml})$ in small volume of urine samples. Limit of detection (LOD) and limit of quantification (LOQ) for the produced fiber were determined $0.25 \mathrm{ng} / \mathrm{ml}$ and $0.825 \mathrm{ng} / \mathrm{ml}$, respectively, and recovery of the fiber was determined $89 \%$ at LOQ. The produced fiber provided a recyclable and solvent free method for extraction of a trace amount of morphine, which can be successfully used for up to 30 times with no significant loss in the extraction efficiency.

\footnotetext{
* Corresponding author: Adel Ghorani-Azam, Medical Toxicology Research Center, Mashhad University of Medical Sciences, Mashhad, Iran, e-mail: adelbiochem@gmail.com Bamdad Riahi-Zanjani, Medical Toxicology Research Center, Mashhad University of Medical Sciences, Mashhad, Iran Mahdi Balali-Mood, Medical Toxicology and Drug Abuse Research Center, Birjand University of Medical Sciences, Birjand, Iran Zarrin Es'haghi, Department of Chemistry, Payame Noor University, 19395-4697, Iran

Ahmad Asoodeh, Department of Chemistry, Faculty of Science, Ferdowsi University of Mashhad, Mashhad, Iran
}

Keywords: HPLC; plant; green chemistry; morphine; nanocomposite; preconcentration; hollow fiber-solid phase microextraction (HF-SPME)

\section{Introduction}

All drugs are metabolized in the body and most of them have half-life of less than $72 \mathrm{~h}$, so there is a limited time for detection of the drugs in biological samples [1]. Also, since the considerable forensic decisions are made on the basis of the levels of analytes in biological samples, accuracy of the detection method in appropriate time is critical [2]. There are numerous confirmatory methods for detection of analytes in biological samples such as HPLC, and GC-mass. Preconcentration of the analytes during conventional sample preparation is considered as an essential part of analyte detection in these methods [3]. However, sample preparation has some drawbacks such as high solvent use and subsequent environmental damage. Preconcentration methods with good efficiency such as microextraction techniques have been developed for detection of small amount of analytes. Solid phase microextraction (SPME) method is considered as a method of desire for extraction of small amount of abused drugs [4,5].

Although microextraction techniques may enhance the sample preparation methods, they are usually associated with some disadvantage such as noncompatibility with biological system [6,7]. Many of the chemicals used in the microextraction methods are not biodegradable and may be dangerous and cause environmental damage [8]. Plants extracts and herbal products are generally considered as a promising therapeutic option for treatment of various diseases $[9,10]$. But in recent years, it is believed that herbal products can be used as sorbent in diagnostic medicine and biosensor technology. In this regards, it is now believed that using environmentally safe method such as plant-based sorbent may enhance the safety of sample preparation as well as the efficiency of extraction method in analytical sciences. 
Recently, plant extract or other herbal products have attracted attentions for use in diagnostic purposes. Since, tobacco extracts contain numerous types of active constituents such as terpenes, alcohols, and polyphenols with different functional groups, including carboxylic acids, and nitrogen-containing compounds, it is expected that morphine can efficiently bind to the nanofiber functionalized with tobacco extract. Hence, in this study, we aimed to develop a SPME fiber for preconcentration and detection of morphine in urine sample using tobacco extract as the sorbent. In addition, we aimed to develop a green and solvent free method for preconcentration of morphine without any time-consuming sample preparation.

\section{Materials and methods}

\subsection{Chemicals and apparatus}

Chemicals including acetone, N,N-Dimethylformamide (DMF), 1-octanol, and acetonitrile (ACN) were purchased from Merck (Darmstadt, Germany). Morphine was obtained from a collaborator laboratory of Mashhad University of medical sciences, Mashhad, Iran. A standard solution of morphine with a concentration of $1000 \mathrm{ng} / \mathrm{ml}$ in deionized water was provided and then stored at $4^{\circ} \mathrm{C}$ until analysis. HPLC-grade water and acetonitrile were purchased from Merck.

A Fourier-transform infrared spectrophotometer (FTIR-8400S, Shimadzu, Japan) and a Raman spectrophotometer (Teksan, Takram P50C0R10, with an excitation wavelength of $532 \mathrm{~nm}$ ) were used to respectively investigate the functional groups of the synthesized nanomaterials and successful modification of the carbon nanotubes (CNTs). To evaluate the structure of the produced fiber in addition to the synthesized nanomaterial, a scanning electron microscope (SEM) (TESCAN MIRA3-FEG, Czech Republic) was used. Moreover, to measure $\mathrm{pH}$ values of the solutions a digital $\mathrm{pH}$ meter with glass electrode (Metrohm-744, Switzerland) was applied. All experiments were performed in triplicate at room temperature $\left(22 \pm 0.5^{\circ} \mathrm{C}\right)$, otherwise it is stated.

\subsection{Extraction of tobacco products}

Tobacco products were extracted by sonication of the tobacco leaves in ultrasonic bath. For this purpose, $50 \mathrm{~g}$ dried tobacco leaves were washed with deionized water and then were sonicated in ultrasonic bath at $60^{\circ} \mathrm{C}$ for $8 \mathrm{~h}$. Afterwards, the extracts were filtered through $0.45 \mu \mathrm{m}$ polytetrafluoroethylene (PTFE) membrane and then lyophilized and kept at $4^{\circ} \mathrm{C}$ for subsequent uses.

\subsection{Synthesis of nanomaterial}

The nanotubes were carboxylated prior to functionalization as previously described [4]. For synthesis of nanomaterial, the carboxylated CNTs were functionalized with the lyophilized tobacco extracts. For this purpose, $20 \mathrm{mg}$ CNTs and $5 \mathrm{mg}$ herbal products (lyophilized tobacco extracts) were dissolved in DMF $(10 \mathrm{ml})$ and sonicated for $1 \mathrm{~h}$ at $60^{\circ} \mathrm{C}$. Subsequently, $10 \mathrm{mg}$ sodium nitrite $\left(\mathrm{NaNO}_{2}\right)$ was added to the solution and sonicated for additional $12 \mathrm{~h}$. The resulted materials were then filtered through $0.45 \mu \mathrm{m}$ PTFE membrane and washed twice with deionized water. Afterwards, the synthesized nanomaterials were dried overnight at $50^{\circ} \mathrm{C}$ under vacuum and used in the study.

\subsection{Preparation of nanomaterial reinforced hollow fiber}

To improve the stability of fibers in addition to other benefits such as simplicity, and selectivity of microextraction technique, hollow fiber (HF) was used for production of SPME fiber. HF is a porous polypropylene fiber with $200 \mu \mathrm{m}$ thickness and the inner diameter of $600 \mu \mathrm{m}$. Hence on structural point of view, the adsorption of analytes may increase in HFs, since adsorption can occur at both inner and outer space of the fiber. For this purpose, HFs were sonicated in acetone for $5 \mathrm{~min}$ to remove the impurities as well as other possible contaminations and then sonicated in octanol for $20 \mathrm{~min}$. The synthesized nanomaterials was added to the solution and sonication was continued for $6 \mathrm{~h}$ at $45^{\circ} \mathrm{C}$, so that the inner side as well as the pores and the surface of HFs were coated with the synthesized nanomaterials functionalized with the tobacco products. The fibers were then dried for use.

\subsection{Sample preparation and HPLC analysis}

For sample preparation, a serial dilution of morphine $(0.1-100 \mathrm{ng} / \mathrm{ml})$ was prepared in urine. For adsorption of the morphine, the fiber was inserted into the sample and sonicated for $5 \mathrm{~min}$. Afterwards, the fiber was washed with $200 \mu \mathrm{l}$ of the washing solution containing water/ methanol (80/20) by sonication for $2 \mathrm{~min}$. To optimize the 


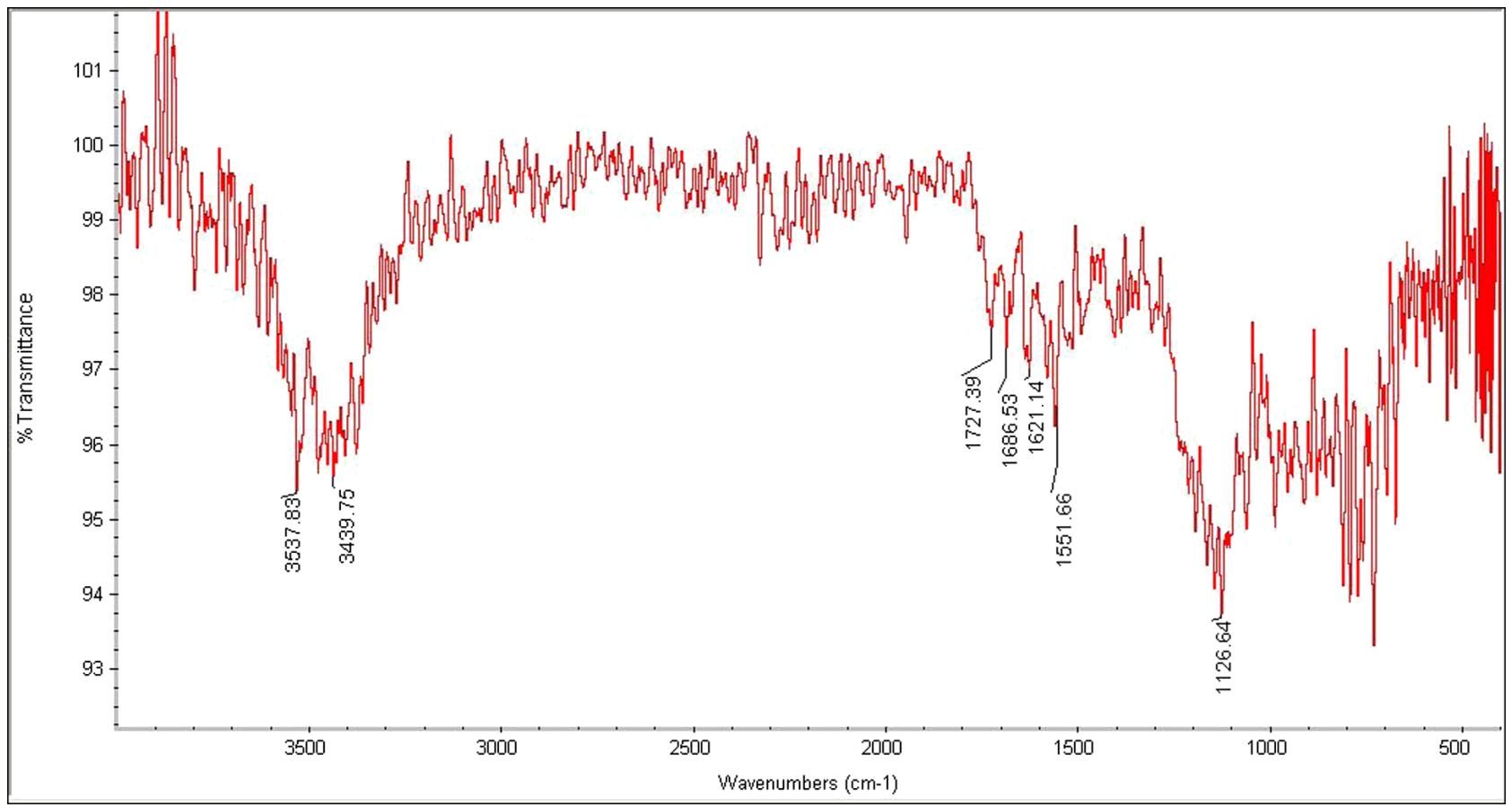

Figure 1: FTIR spectra of the carbon nanotube functionalized with tobacco extract.

microextraction process, functionality of the fiber was evaluated at a range of $\mathrm{pH}(4-12)$ and temperature $\left(10^{\circ} \mathrm{C}\right.$ $\left.50^{\circ} \mathrm{C}\right)$ and in the absence or presence of the salts $(\mathrm{NaCl}$ and $\mathrm{CaCl}_{2}$ ). HPLC (Knauer, Berlin, Germany) system with C18 HPLC column $(10 \times 250 \mathrm{~mm})$ was used to measure the preconcentrated morphine in urine sample. Since the retention times of morphine is 4.3-5 min, HPLC analysis was performed over a period of $10 \mathrm{~min}$ in isocratic mode. Acetonitrile-sodium acetate (10:90, v/v; sodium acetate buffer $0.01 \mathrm{M}$; pH 4) was used as the mobile phase with a flow rate of $0.6 \mathrm{ml} / \mathrm{min}$. According to UV-Visible spectrophotometer measurements, the UV detection wavelength was set at $285 \mathrm{~nm}$.

\section{Results}

\subsection{Characterization and morphology of the synthesized nanocomposite}

Physiochemical properties of the synthesized nanomaterial including successful functionalization and the size of nanomaterials were confirmed by FTIR analysis, Raman measurements and SEM imaging, respectively. Microscopic analysis revealed that the nanocomposites are homogeneously distributed, and functionalized with molecules that have amine, carbonyl, hydroxyl and other functional groups as confirmed by FTIR spectroscopic technique. FTIR spectra showed that functionalization of the synthesized nanocomposite has been successfully completed (Figure 1). The peak at $3537 \mathrm{~cm}^{-1}$ corresponds to $\mathrm{OH}$ group. Amide bound ( $\mathrm{NH}$ stretch) was shown at $3439 \mathrm{~cm}^{-1}$. In addition, a frequency at 1727 was attributed to $\mathrm{C}=\mathrm{O}$ group of amide bound. Also, the peak at $1686 \mathrm{~cm}^{-1}$ is a result of formation of amide ( $\mathrm{C}=\mathrm{O}$ stretch). Raman measurements also confirmed that the structure of CNTs have been modified after carboxylation and functionalization. According to the data of Raman measurements, changes in intensities of the peaks related to the defects in the graphitic structure (D), and tangential vibration of the carbon atoms of the nanotube (G) indicated modification of CNTs (Figure 2). SEM images also showed the homogenous structure of the nanocomposite and microstructure of the fiber (Figures $3 a$ and $3 b)$.

\subsection{Detection of morphine in urine sample and optimization of microextraction process}

For detection of the preconcentrated morphine by HPLC, $15 \mu \mathrm{l}$ of the solution was injected into HPLC system. HPLC 


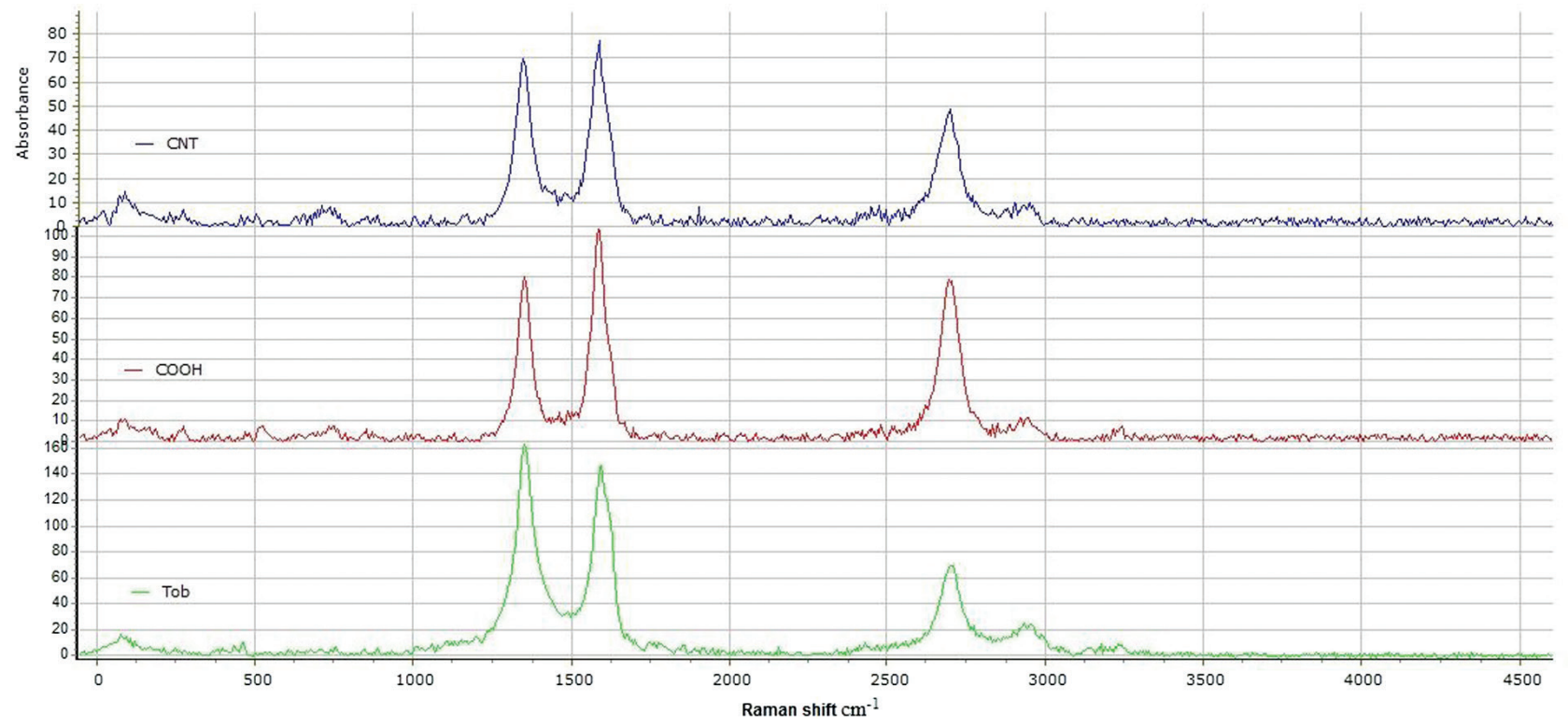

Figure 2: Raman spectra of the raw CNT, carboxylated CNT and tobacco-functionalized CNT.

data showed that, without microextraction process, morphine could not be detected in urine samples containing lower concentration of morphine $(\leq 10 \mathrm{ng} / \mathrm{ml})$. However, microextraction with the produced fiber could concentrate the morphine by more than 20 -fold, since $0.25 \mathrm{ng} / \mathrm{ml}$ of morphine in urine sample was also detectable after microextraction.

The efficiency of microextraction was evaluated at different environmental conditions including temperature, $\mathrm{pH}$, extraction time, and the presence of salts. The results showed that the optimum condition for microextraction was $5 \mathrm{~min}$ at $40^{\circ} \mathrm{C}$ in sonication bath (Figures $4 \mathrm{a}$ and $4 \mathrm{~b}$ ). On the other hand, our findings showed that salt such as $\mathrm{NaCl}$, and $\mathrm{CaCl}_{2}$ reduces the efficiency of microextraction process (Figure 4c). The results also showed that the morphine can more efficiently bind to the fiber at neutral $\mathrm{pH}$ of around $\mathrm{pH}=6$ (Figure 4d). In addition, the prepared nanofiber showed a suitable stability at the defined conditions (optimum $\mathrm{pH}$, temperature, time of extraction, and without salt), where the fiber was used for 30 times with no considerable loss in its extraction efficiency.

\subsection{Validation of the produced fiber in real sample}

To investigate the validity of the produced fiber performance in real samples, morphine-positive urine samples from patients admitted to the poisoned patients department of Imam Reza hospital, Mashhad, Iran, which had been subjected to be discarded were collected and applied to morphine detection. Findings confirmed that the produced fiber can preconcentrate the morphine in urine samples (Figures $5 \mathrm{a}$ and $5 \mathrm{~b}$ ). The results also demonstrated that adsorption of morphine to the fiber in morphine-positive urine samples tracks a linear range at two levels $(0.25-10 \mathrm{ng} / \mathrm{ml}$ and $10-1000 \mathrm{ng} / \mathrm{ml})$. Furthermore, limit of quantification (LOQ) and the limit of detection (LOD) were found to be 0.825 and $0.25 \mathrm{ng} / \mathrm{ml}$, respectively. Recovery of the fiber was also measured $89 \%$ at LOQ level.

\section{Discussion}

Detection of small amount of analytes in biological samples is of great importance particularly in forensic medicine. This is particularly important when only a small amount of analyte is available in biological sample due to taking a low-dose of the drugs or even passing a long time after the last taking of the medication $[11,12]$. Extraction and preconcentration is promising approach for detection of these trace analytes; however, this method has some shortcoming such as non-biodegradability and non-biocompatibility with the environment. Moreover, a comprehensive global effort has been recently started to reduce hazardous wastes of chemical origin in addition to developing 


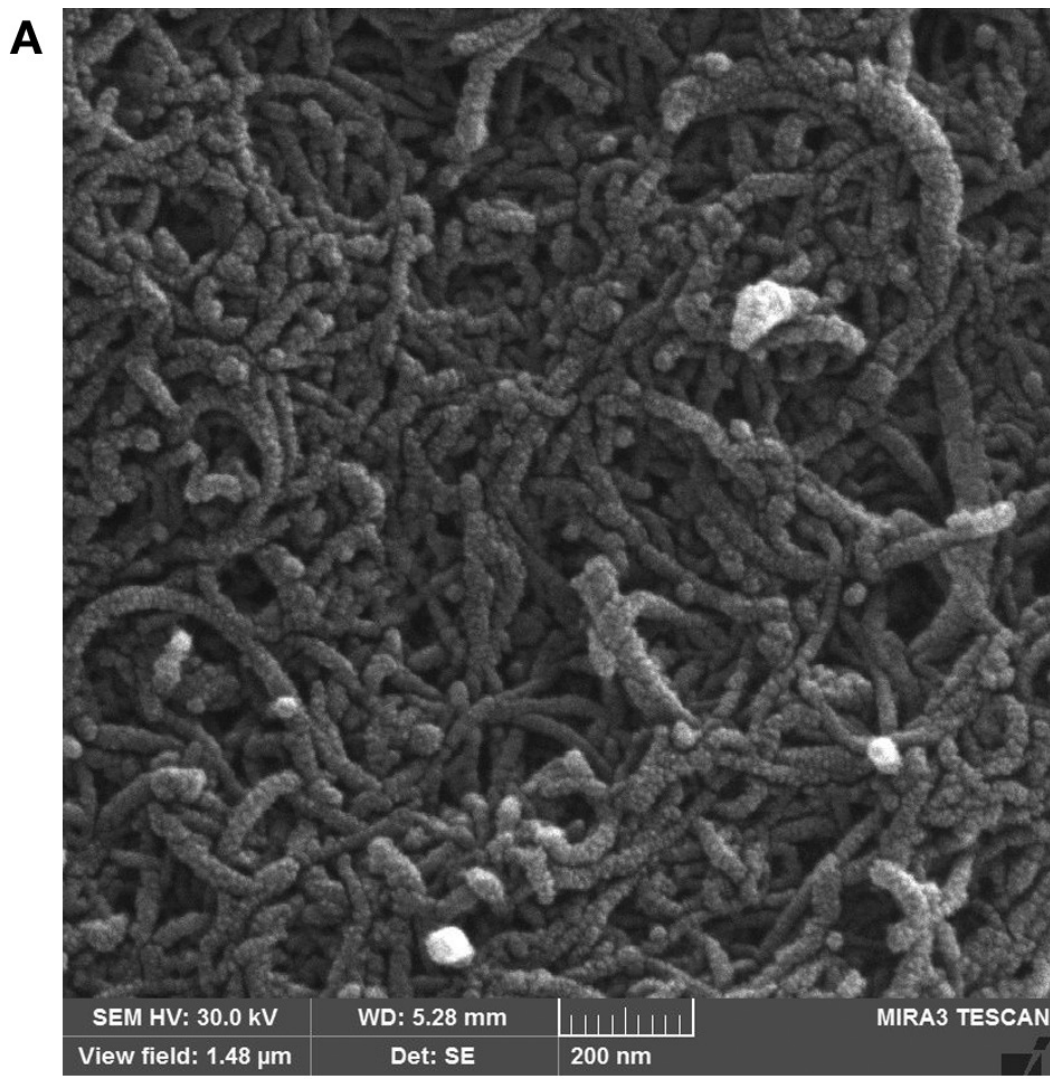

B

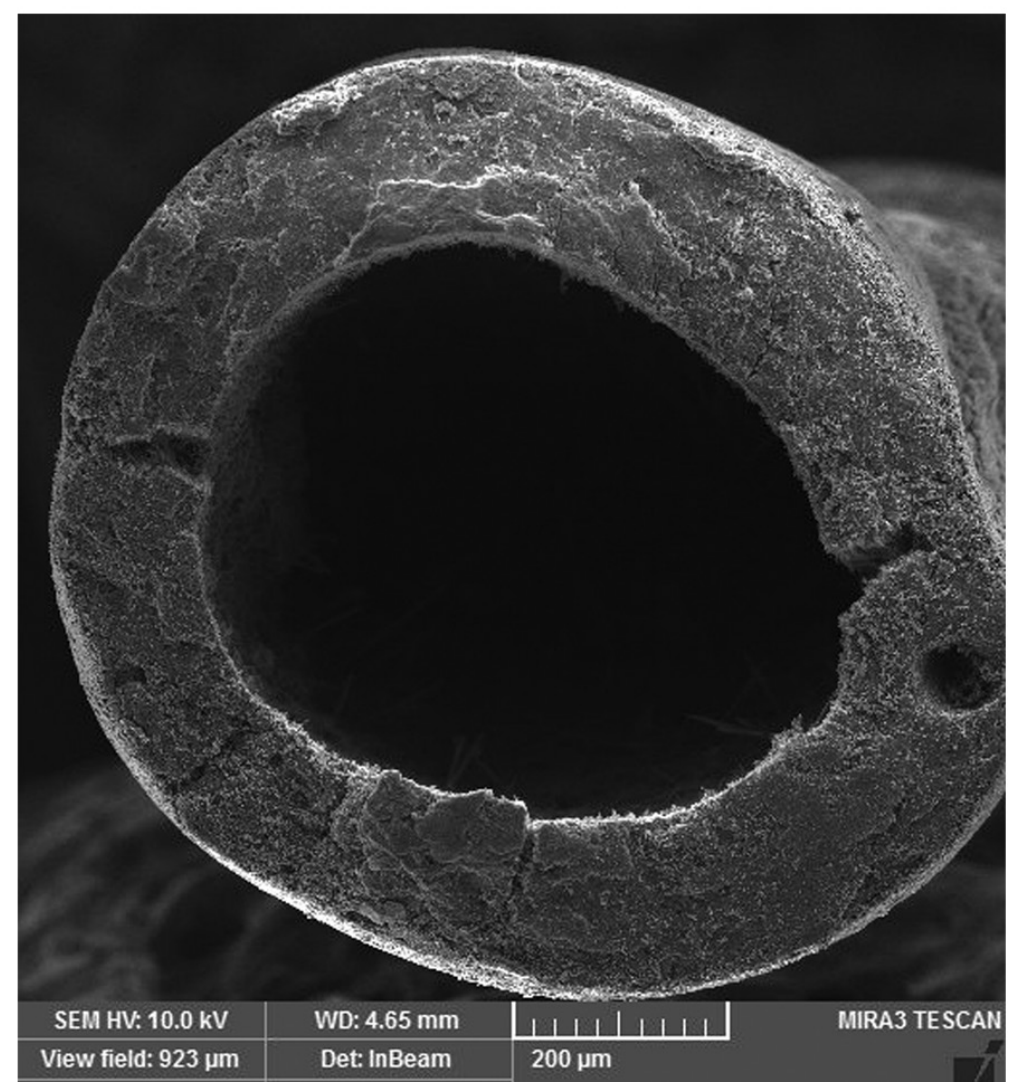

Figure 3: SEM image of the carbon nanotube functionalized with tobacco extract (a), and microstructure of the hallow fiber (b). 

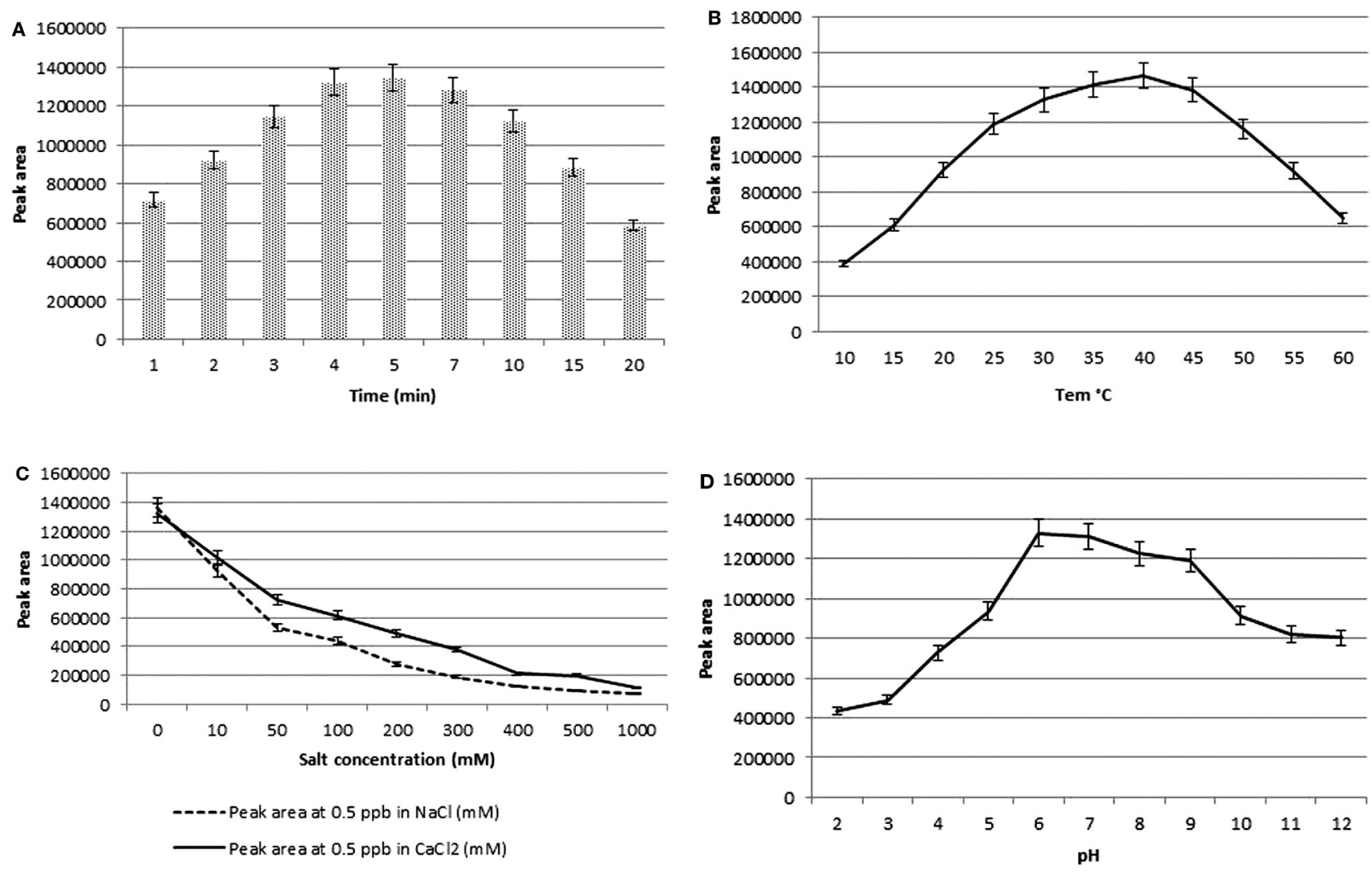

Figure 4: Effect of time (a), temperature (b), salts (c), and pH (d) on microextration efficiency of the SPME fiber.

economical, cost-effective, and safe method for analysis of chemicals and toxicants in the reference laboratories as well as in clinical practices for diagnostic purposes [13]. In addition, to overcome the disadvantages of routine microextraction technique, recent studies have focused on developing green methods with the lowest solvent use as possible using biocompatible nanomaterials [14]. For this reason, plant extracts or other purified herbal products are the era of interest for many researchers in green chemistry or diagnostic medicine, and recent findings showed that herbal products or plant extract can be used at different stages of method development $[15,16]$. In the present study, tobacco extracts containing different products with various functional groups were used as sorbent of morphine in the sample. The use of plant extract as a selective sorbent for microextraction and preconcentration of cadmium as a trace analyte in the water and fruit samples had been previously reported [17]. Also, the advantages of green extraction method using herbal products over chemical methods have been emphasized. Theoretically, the main purpose of green extraction method is developing new extraction processes or new alternative solvents using renewable and biodegradable plant resources to minimize hazardous chemical wastes [18]. Some of the most important advantages of plant-based analyte extraction in comparison with conventional chemical methods include fewer environmental damages due to fewer solvent use, cost-effective procedure especially in mass production, and no further necessity to use high temperature, pressure, and other hazardous chemicals during preparation of the fiber [19].

Nowadays, plant-based extraction techniques are used for extraction and preconcentration of highquality natural products from plant in addition to their byproducts [20-22]. It has been reported that biodegradable aminophenol-functionalized resin can be used for green determination and preconcentration of metal ions including copper $(\mathrm{Cu})$, zinc $(\mathrm{Zn})$, lead $(\mathrm{Pb})$ and cadmium (Cd) in drinking tap water [23]. Solid phase extraction and preconcentration method using a natural sorbent based on cotton roll dyed by quinalizarin has also been successfully applied for determination of 14 rare earth elements as well as uranium with acceptable accuracy, precision and significant enrichment factor [24]. Other environmental friendly method has also been successfully used for preconcentration and 

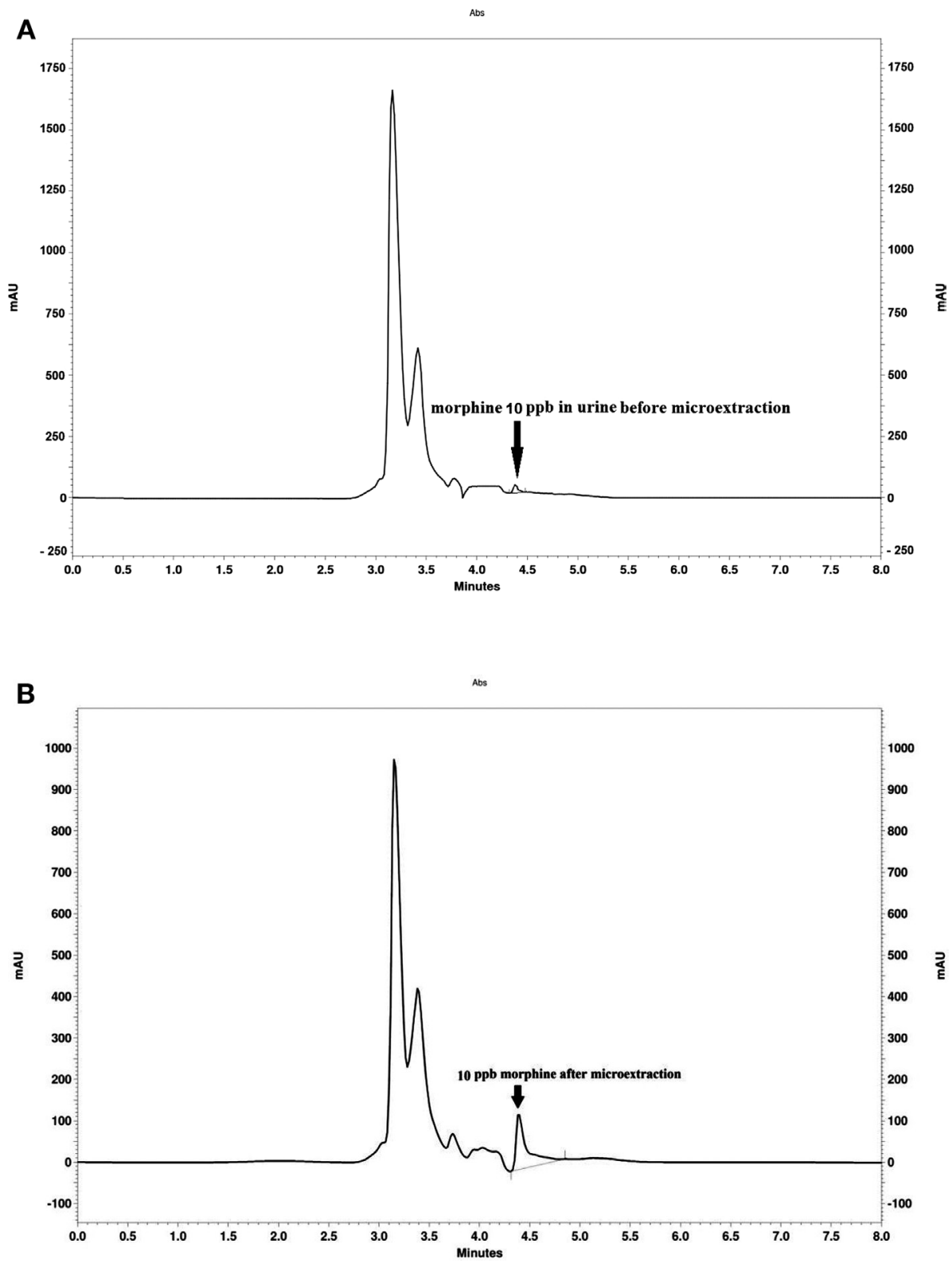

Figure 5: HPLC chromatograms of the morphine preconcentrated with the SPME method. The chromatograms show morphine peak before (a) and after microextraction (b).

simultaneous determination of cobalt $(\mathrm{Co})$ and lead $(\mathrm{Pb})$ in waste water sample using 8-hydroxyquinoline (oxine) as a chelating agent [25].

So far, plant extract or herbal products have not been used as sorbent for separation or analytical purpose. In the present study, a new SPME fiber was designed and produced that was recyclable after the extraction process is completed. Also, since non-chemical agents of plant origin was used for preparation of the fiber, it can be considered as less hazardous to the environment as well as the operators. Findings showed that the produced fiber could efficiently adsorb the morphine in the water and urine samples. Specificity of the fiber to the morphine was relatively high, since by increasing the concentration of morphine in the urine sample, morphine molecules were more successful in competition with other analytes present in the urine. This could be partly due to higher binding affinity of morphine to the surface sorbent of the produced fiber. Findings of the current research may help to develop a new green and solvent-free method for extraction of analytes, especially drugs of abuse. 


\section{Conclusion}

Findings of the current study suggested that the provide method could improve the efficiency of morphine detection. In addition, the described method is a green analytical method with the lowest use of chemical solution in which a biodegradable fiber could be used for several times with no significant loss in concentrating capacity.

Acknowledgment: The results described in this paper were parts of $\mathrm{PhD}$ thesis in toxicology, supported by the Vice Chancellor for Research of Mashhad University of Medical Sciences with grant No: 930829.

Conflict of interest: There is no conflict of interest between the authors.

\section{References}

[1] Fernandez E., Perez R., Hernandez A., Tejada P., Arteta M., Ramos J.T., Factors and Mechanisms for Pharmacokinetic Differences between Pediatric Population and Adults. Pharmaceutics, 2011, 3, 53-72.

[2] Gidal B.E., Clark A.M., Anders B., Gilliam F., The application of half-life in clinical decision making: Comparison of the pharmacokinetics of extended-release topiramate (USL255) and immediate-release topiramate. Epilepsy. Res., 2017, 129, 26-32.

[3] Lum T.-S., Tsoi Y.-K., Leung K.S.-Y., Current developments in clinical sample preconcentration prior to elemental analysis by atomic spectrometry: a comprehensive literature review. J. Anal. At. Spectrom., 2014, 29, 234-241.

[4] Riahi-Zanjani B., Balali-Mood M., Asoodeh A., Es'haghi Z., Ghorani-Azam A., Developing a new sensitive solidphase microextraction fiber based on carbon nanotubes for preconcentration of morphine. Appl. Nanosci., 2018, DOI:10.1007/s13204-018-0882-x.

[5] Riahi-Zanjani B., Balali-Mood M., Asoodeh A., Es'haghi Z., Ghorani-Azam A., Potential application of amino acids in analytical toxicology. Talanta, 2019, 197, 168-174.

[6] Rutkowska M., Dubalska K., Konieczka P., Namiesnik J., Microextraction techniques used in the procedures for determining organomercury and organotin compounds in environmental samples. Molecules, 2014, 19, 7581-7609.

[7] Sharifi V., Abbasi A., Nosrati A., Application of hollow fiber liquid phase microextraction and dispersive liquid-liquid microextraction techniques in analytical toxicology. J. Food. Drug. Anal., 2016, 24, 264-276.

[8] Ghorani-Azam A., Riahi-Zanjani B., Balali-Mood M., Effects of air pollution on human health and practical measures for prevention in Iran. J. Res. Med. Sci., 2016, 21, 65.

[9] Sepahi S., Ghorani-Azam A., Sepahi S., Asoodeh A., Rostami S., In Vitro Study to Evaluate Antibacterial and Non-haemolytic Activities of Four Iranian Medicinal Plants. West. Indian. Med. J., 2014, 63, 289-293.

[10] Ghorani-Azam A., Sepahi S., Khodaverdi E., Mohajeri S.A., Herbal medicine as a promising therapeutic approach for the management of vascular dementia: A systematic literature review. Phytother. Res., 2018, 32, 1720-1728.

[11] Riahi-Zanjani B., Balali-Mood M., Es'haghi Z., Asoodeh A., Ghorani-Azam A., Molecular modeling and experimental study of a new peptide-based microextraction fiber for preconcentrating morphine in urine samples. J. Mol. Model., 2019, 25.

[12] Riahi-Zanjani B., Balali-Mood M., Aflatoxin M1 contamination in commercial pasteurized milk from local markets in Fariman, Iran. Mycotoxin. Res., 2013, 29, 271-274.

[13] Armenta S., Garrigues S., de la Guardia M., The role of green extraction techniques in Green Analytical Chemistry. Trends. Analyt. Chem., 2015, 71, 2-8.

[14] Mohseni S., Aghayan M., Ghorani-Azam A., Behdani M., Asoodeh A., Evaluation of antibacterial properties of Barium Zirconate Titanate (BZT) nanoparticle. Braz. J. Microbiol., 2014, 45, 1393-1399.

[15] Jain S., Mehata M.S., Medicinal Plant Leaf Extract and Pure Flavonoid Mediated Green Synthesis of Silver Nanoparticles and their Enhanced Antibacterial Property. Sci. Rep., 2017, 7, 15867.

[16] Ahmed S., Ahmad M., Swami B.L., Ikram S., A review on plants extract mediated synthesis of silver nanoparticles for antimicrobial applications: A green expertise. J. Adv. Res., 2016, 7, 17-28.

[17] Mehdinia A., Shegefti S., Shemirani F., A novel nanomagnetic task specific ionic liquid as a selective sorbent for the trace determination of cadmium in water and fruit samples. Talanta, 2015, 144, 1266-1272.

[18] Chemat F., Vian M.A., Cravotto G., Green extraction of natural products: concept and principles. Int. J. Mol. Sci., 2012, 13, 8615-8627.

[19] Dhuper S., Panda D., Nayak P., Green synthesis and characterization of zero valent iron nanoparticles from the leaf extract of Mangifera indica. Nano Trends: J. Nanotech. App., 2012, 13, 16-22.

[20] Ameer K., Shahbaz H.M., Kwon J.H., Green Extraction Methods for Polyphenols from Plant Matrices and Their Byproducts: A Review. Compr. Rev. Food. Sci. Food. Saf., 2017, 16, 295-315.

[21] Giancarlo C., Arianna B., Laura O., Green extraction techniques for high-quality natural products. Agro. Food. Ind. Hi. Tech., 2011, 22, 24-36. 
[22] Chen Y., Du K., Li J., Bai Y., An M., Tan Z., et al., A Green and Efficient Method for the Preconcentration and Determination of Gallic Acid, Bergenin, Quercitrin, and Embelin from Ardisia japonica Using Nononic Surfactant Genapol X-080 as the Extraction Solvent. Int. J. Anal. Chem., 2018, 2018, 1707853.

[23] Burham N., Azeem S.A., El-Shahat M.F., Solid phase selective separation and green preconcentration of $\mathrm{Cu}, \mathrm{Zn}, \mathrm{Pb}$ and $\mathrm{Cd}$ in drinking water by using novel functionalized resin. Cent. Eur. J. Chem., 2009, 7, 945.
[24] Zari N., Hassan J., Tabar-Heydar K., Ahmadi S.H., On-line Green Solid Phase Extraction of Trace Rare Earth Elements and Uranium in Environmental Samples and ICP OES Detection. J. Braz. Chem. Soc., 2016, 27, 1881-1888.

[25] Naeemullah, Kazi T.G., Shah F., Afridi H.I., Khan S., Arian S.S., et al., A green preconcentration method for determination of cobalt and lead in fresh surface and waste water samples prior to flame atomic absorption spectrometry. J. Anal. Methods. Chem., 2012, 2012, 713862. 